\title{
On decomposable mappings of manifolds
}

\author{
By \\ Yoshihiro SAITO
}

(Received April 19, 1962)

In the present paper we study some relations between the singularities of mappings and the decomposable mappings of manifolds. Throughout this paper by a smooth mapping (function) we understand a $C^{\infty}$-mapping $\left(C^{\infty}\right.$-function); $M^{n}$ denotes an orientable closend $n$-dimensional $C^{\infty}$-manifold, and $R^{n}$ the $n$-dimensional Euclidean space.

We shall now recall briefly the definitions of the singularities $S_{r}(f), S_{r, r^{\prime}}(f), \cdots$ of a mapping $f: M^{n} \rightarrow R^{p}, n \geqq p$, [3], [5].

Let $S_{r}(f)$ denote the set of points of $M^{n}$ at which $f$ has rank $p-r$. Suppose that $S_{r}(f)$ is an $m$-dimensional submanifold of $M^{n}$. Then $S_{r, r^{\prime}}(f)$ is defined to be the subset of $S_{r}(f)$ consisting of points at which the mapping $f$ restridted on $S_{r}(f)$ has rank $m-r^{\prime}$. By the similar way we define the singularities $S_{r, r^{\prime}, \cdots, r^{\prime \prime}}(f)$.

We shall give a condition under which $S_{r}(f)$ is a submanifold of $M^{n}$. Let $G(f)$ be the graph of $f$, and associate to each point $p$ of $M^{n}$ the tangent space of $G(f)$ at $(p, f(p))$. Then we have a mapping, denoted by $d_{1} f$, of $M^{n}$ to $B_{0}$, the space of $n$-planes in the tangent spaces of $M^{n} \times R^{p}$.

$B_{0}$ is a fibre bundle over $M^{n} \times R^{p}$ whose fibre is the Grassmann manifold $G_{n}^{p}$, the space of $n$-planes in $R^{n+p}$. Denote $B_{1}=\bigcup_{q} F_{r}(q)$ where $F_{r}(q)=(p-r, \cdots, p-r, p, \cdots, p)$ is the Schubert variety in the fibre $G_{n}^{p}(q)$ over a point $q$ of $M^{n} \times R^{p}$. Then $B_{1}$ is a submanifold of $B_{0}$. Since we have $S_{r}(f)=d_{1} f^{-1}\left(B_{1}\right)$, it follows that if the mapping $d_{1} f$ is $t$-regular (transverse regular) on $B_{1}$ then $S_{r}(f)$ is a regular submanifold of $M^{n}$.

Now we suppose that $d_{1} f$ is $t$-regular on $B_{1}$. Let $m$ be the 
dimension of $S_{r}(f)$, and let $H(q)$ denote the set of $m$-planes which are contained in $n$-planes regarded as points of the $F_{r}(q)$. Then $H=\bigcup_{q \in B_{1}} H(q)$ is a fibre bundle over $B_{1}$ and its fibre is $G_{m}^{n-m}$. Define $d_{2} f$ to be a mapping such that $d_{2} f(p)$ is the tangent space of $S_{r}(f)$ at $p$. For an intger $r^{\prime} \leqq p-r$ and a point $q^{\prime} \in B_{1}$ projected to $q$, we denote by $F_{r^{\prime}}^{\prime}\left(q^{\prime}\right)$ the set of $m$-planes $V^{m}\left(q^{\prime}\right) \subset V^{n}(q)$ which are projected to $R^{p}$ with rank $p-r-r^{\prime}$. Denote $B_{2}=\bigcup_{q^{\prime}} F_{r^{\prime}}^{\prime}\left(q^{\prime}\right)$ where $q^{\prime} \in B_{1}$. Then $B_{2}$ is a submanifold of $H$ and $S_{r, r^{\prime}}(f)=d_{2} f^{-1}\left(B_{2}\right)$. Thus it follows that if $d_{2} f$ is $t$-regular on $B_{2}$ then $S_{r, r^{\prime}}(f)$ is a submanifold of $S_{r}(f)$. Furthermore we can obtain the similar conditions under which the singularities of a mapping are regular submanifolds. A mapping satisfying these conditions is called generic mapping.

Let $\rho: R^{n+1} \rightarrow R^{n}$ denote the projection, and let $f$ be a mapping of $M^{n}$ to $R^{n}$. Then if there exists an immersion $i: M^{n} \rightarrow R^{n+1}$ satisfying $f=\rho i$ we say that $f$ is decomposable [1].

Given mappings $f, \bar{f}: M^{n} \rightarrow R^{p}$, if the $r$-th partial derivatives of $f$ and $\bar{f}$ are sufficiently close for all $r \leqq s$, we say that $\bar{f}$ is a good $s$-approximation of $f$.

Our main results in this paper are stated as follows.

Theorem 3. Let $M^{3}$ be an orientable closed smooth 3-manifold and $f$ be a smooth mapping of $M^{3}$ to $R^{3}$. Suppose that $f$ is a generic debomposable mapping. Then we may take a good 0-approximation $\vec{f}$ of $f$ so that $S_{1,1}(\bar{f})=$ the empty set $\varnothing$.

Theorem 4. Let $M^{n}$ be an orientable closed smooth n-manifold and $f$ be a generic mapping of $M^{n}$ to $R^{n}$. Suppose that the singularities of $f$ satisfy the following conditions.

$$
S_{i}(f)=\varnothing \quad i \geqq 2, \quad S_{1,1}(f)=\varnothing .
$$

Then the mapping $f$ is a decomposable mapping.

1. We shall now consider the case for $n=p=3$. In this case it is well-known [5] that $S_{i}(f)=\varnothing$ for any generic mapping $f$ and $i \geqq 2$. Hence we may consider only $S_{1}(f), S_{1,1}(f)$ and $S_{1,1,1}(f)$. Given a point $q \in M^{3}$, we may take (local) coordinatate systems $(x, y, z)$ 
at $q$ and $(X, Y, Z)$ at $f(q)$ in which $f$ is represented by $X=x, Y=y$ $Z=h(x, y, z)$. Then the tangent space of the graph $G(f)$ is represented as follows:

$$
X^{\prime}-x^{\prime}=0, Y^{\prime}-y^{\prime}=0 \text { and } Z^{\prime}-\frac{\partial h}{\partial x} x^{\prime}-\frac{\partial h}{\partial y} y^{\prime}-\frac{\partial h}{\partial z} z^{\prime}=0
$$

where $\left(x^{\prime}, y^{\prime}, z^{\prime}\right),\left(X^{\prime}, Y^{\prime}, Z^{\prime}\right)$ are the bases of the tangent spaces.

Hence $\frac{\partial h}{\partial z}=0$ if and only if the rank of the projection of the tangent space of $G(f)$ to $R^{3}$ is 2 . Thus the set $S_{1}(f)$ is represented by $\frac{\partial h}{\partial z}=0$. In this case the normal coordinate of $F_{1}$ is given by $\frac{\partial h}{\partial z}$. This shows that a condition for the $t$-regularity of $d_{1} f$ on $B_{1}$ is that at least one of the derivatives $\frac{\partial^{2} h}{\partial x \partial z}, \frac{\partial^{2} h}{\partial y \partial z}$ and $\frac{\partial^{2} h}{\partial z^{2}}$ is not zero.

Next we shall consider $S_{1,1}(f)$. The tangent space of $S_{1}(f)$ is represented by the equation (1) and

$$
\frac{\partial^{2} h}{\partial x \partial z} x^{\prime}+\frac{\partial^{2} h}{\partial y \partial z} y^{\prime}+\frac{\partial^{2} h}{\partial z^{2}} z^{\prime}=0
$$

Hence $\frac{\partial h}{\partial z}=\frac{\partial^{2} h}{\partial z^{2}}=0$ if and only if the rank of the projection of the tangent space of $S_{1}(f)$ to $R^{3}$ is 1 . Therefore the set $S_{1,1}(f)$ is represented by $\frac{\partial h}{\partial z}=\frac{\partial^{2} h}{\partial z^{2}}=0$. In this case, the normal coordinate of $F_{1}^{\prime}$ is given by $\frac{\partial^{2} h}{\partial z^{2}}$. Let $\left(z^{\prime}, s^{\prime}\right)$ denote a basis of the tangent space of $S_{1}(f)$. Then a condition for the $t$-regularity of $d_{2} f$ on $B_{2}$ is that at least one of the derivatives $\frac{\partial^{3} h}{\partial s \partial z^{2}}$ and $\frac{\partial^{3} h}{\partial z^{3}}$ is not zero.

The tangent space of $S_{1,1}(f)$ is represented by the equations (1), (2) and

$$
\frac{\partial^{3} h}{\partial x \partial z^{2}} x^{\prime}+\frac{\partial^{3} h}{\partial y \partial z^{2}} y^{\prime}+\frac{\partial^{3} h}{\partial z^{3}} z^{\prime}=0 .
$$

Note that $S_{1,1,1}(f)$ is the set of points $q \in S_{1,1}(f)$ such that the tangent line of $S_{1,1}(f)$ at $q$ is projected to $R^{3}$ with rank 0 . Therefore the set $S_{1,1,1}(f)$ is represented by the following equations 


$$
\begin{aligned}
& \frac{\partial h}{\partial z}=\frac{\partial^{2} h}{\partial z^{2}}=\frac{\partial^{3} h}{\partial z^{3}}=0, \\
& \frac{\partial^{2} h}{\partial x \partial z} \frac{\partial^{3} h}{\partial y \partial z^{2}}-\frac{\partial^{2} h}{\partial y \partial z} \frac{\partial^{3} h}{\partial x \partial z^{2}}=0 .
\end{aligned}
$$

Since $\frac{\partial^{3} h}{\partial z^{3}}$ is a normal coordinate of $F_{1}^{\prime \prime}$, the mapping $d_{3} f$ is $t$-regular on $F_{1}^{\prime \prime}$ if and only if $\frac{\partial^{4} h}{\partial z^{4}} \neq 0$.

2. The types of singularities. In this section we suppose that mapping $f$ of $R^{3}$ to $R^{3}$ is generic and it maps the origin 0 to the origin 0 . The singularities of $f$ are divided into three types $S_{1}, S_{1,1}$ and $S_{1,1,1}$.

Case 1 ( 0 is a point of $S_{1}(f)-S_{1,1}(f)$ ). We may take coordinate systems in which $f$ is represented by

$$
X=x, Y=y \text { and } Z=h(x, y, z) .
$$

Since 0 is a point of $S_{1}(f)$ the Taylor expansion of $h$ does not contain the constant term and terms of the first order. Therefore we have

$$
Z=a(x, y)+a_{13} x z+a_{23} y z+a_{33} z^{2}+R, \text { ord }{ }_{z} R \geqq 3^{1)} .
$$

Set

$$
x^{\prime}=x, y^{\prime}=y, z^{\prime}=z, X^{\prime}=X, Y^{\prime}=Y \text { and } Z^{\prime}=Z-a(X, Y) .
$$

This gives, dropping primes,

$$
X=x, Y=y, Z=a_{13} x z+a_{23} y z+a_{33} z^{2}+R .
$$

Since $f$ is generic, at least one of $a_{13}, a_{23}$ and $a_{33}$ is not zero. Further the origin is not the point of $S_{1,1}(f)$, therefore the tangent space of $S_{1}(f)$ at 0 is transversal to the null space $N(0)$ of $f^{2}$. $S_{1}(f)$ is now represented by the following equation

$$
a_{13} x+a_{23} y+2 a_{33} z+R_{z}=0^{3)},
$$

1) $\operatorname{ord}_{z} R$ denotes the order of $R$ with respect to the variable $z$.

2) $N(p)$ denotes the null space of $f$, the linear subspace of the tangent space which is mapped to zero vector by the differential of $f$.

3) $R_{z}$ denotes the first partial derivative of $R$ with respect to $z$. 
and hence the equation of the tangent plane of $S_{1}(f)$ at 0 is $a_{13} x+a_{23} v+2 a_{33} z=0$. Since this plane does not contain the $z$-axis, we have $a_{33} \neq 0$. If $a_{13} \neq 0$ or $a_{23} \neq 0$ then we may represent $f$ in suitable coordinates as follows:

$$
X=x, Y=y, Z=x z+a_{33} z^{2}+R, \text { ord }_{z} R \geqq 3 .
$$

Set

$$
\begin{aligned}
& x=x^{\prime}, y=y^{\prime}, z=z^{\prime}-\frac{x^{\prime}}{2 a_{33}}, X=X^{\prime}, Y=Y^{\prime} \\
& \text { and } Z=a_{33} Z^{\prime}+\left(\frac{1-2 a_{33}}{4 a_{33}^{2}}\right) X^{\prime 2} .
\end{aligned}
$$

Then we have, dropping primes,

$$
X=x, Y=y, Z=z^{2}+R, \text { ord } R \geqq 3 .
$$

Case 2 (0 is a point of $\left.S_{1,1}(j)-S_{1,1,1}(f)\right)$. In this case the expansion of $h$ does not contain the term $z^{2}$, because 0 belongs to $S_{1,1}(f)$. Hence $f$ is represented in a new coordinates as follows:

$$
X=x, Y=y, Z=y z+R, \text { ord } R \geqq 3 .
$$

Since the expansion of $h$ contains $y z$, we may omit in the expansion of $R$ the terms which contain $z$ with at most order 1 . Then the formulas for $X, Y$ and $Z$ becoms as follows in a new coordinate systems :

$$
X=x, Y=y, Z=y z+a_{133} x z^{2}+a_{233} y z^{2}+a_{333} z^{3}+R, \text { ord } R \geqq 4 .
$$

Set $z^{\prime}=z+a_{233} z^{2}$ then we have, $z=z^{\prime}+\varphi\left(z^{\prime}\right)$ for small $z^{\prime}$ where ord $\mathscr{P} \geqq 2$. Therefore we have, dropping primes,

$$
X=x, Y=y, Z=y z+a_{133} x z^{2}+a_{333} z^{3}+R, \text { ord } R \geqq 4 .
$$

Then the equation of $S_{1,1}(f)$ are represented as follows:

$$
\begin{aligned}
& y+2 a_{133} x z+3 a_{333} z^{2}+R_{z}=0, \\
& 2 a_{133} x+6 a_{333} z+R_{z z}=0 .
\end{aligned}
$$

Hence the tangent line of $S_{1,1}(f)$ at 0 is represented by the following equations :

$$
y=0, a_{133} x+3 a_{333} z=0 .
$$


Since $0 \notin S_{1,1,1}(f)$, the tangent line of $S_{1,1}(f)$ at 0 does not coincide with the $z$-axis. Hence we have $a_{333} \neq 0$.

Set

$$
\begin{aligned}
x^{\prime} & =\frac{x}{\sqrt[3]{a_{333}^{2}}}, y^{\prime}=\frac{y}{-\sqrt[3]{a_{333}}}, z^{\prime}=-\sqrt[3]{a_{333}} z, \\
X^{\prime} & =\frac{X}{\sqrt[3]{a_{333}^{2}}}, Y^{\prime}=\frac{Y}{-\sqrt[3]{a_{333}}}, Z^{\prime}=Z
\end{aligned}
$$

then we have, dropping primes,

$$
X=x, Y=y, Z=y z+a_{133} x z^{2}-z^{3}+R, \text { ord } R \geqq 4 .
$$

If $a_{133} \neq 0$, we set $z^{\prime}=z+\frac{a_{133}}{3} x$. Then we have, dropping primes,

$$
X=x, Y=y, Z=y z-z^{3}+R, \text { ord } R \geqq 4 .
$$

Case (0 is a point of $S_{1,1,1}(f)$ ) By the same reason as in case 2 , the expansion of $h$ becomes

$$
Z=x z+a_{233} y z^{2}+a_{333} z^{3}+R, \text { ord } R \geqq 4 .
$$

Since 0 is a point of $S_{1,1,1}(f)$, we have, $a_{333}=0$. Since $f$ is generic, this implies $a_{233} \neq 0$.

Set $y^{\prime}=a_{233} y$ and $Y^{\prime}=a_{233} Y$, then we have, dropping primes,

$$
X=x, Y=y, Z=x z+y z^{2}+R, \text { ord } R \geqq 4 .
$$

The last equation contains $x z$ and $y z^{2}$, and so we may omit in $R$ the terms of fourth order which contain $z$ with at most order 2 .

Then we have

$$
Z=x z+y z^{2}+a x z^{3}+b y z^{3}+c z^{4}+R, \text { ord } R \geqq 5 .
$$

Set $z^{\prime}=z+a z^{3}$, then we have $z=z^{\prime}+q\left(z^{\prime}\right)$ for small $z^{\prime}$, where ord $\rho \geqq 3$. This coordinate transformation gives us, dropping primes,

$$
Z=x z+y z^{2}+b y z^{3}+c z^{4}+R, \text { ord } R \geqq 5 .
$$

Now the set $S_{1}(f)$ and $S_{1,1}(f)$ are represented by the following equations : 


$$
\begin{gathered}
S_{1}(f): F(x, y, z)=x+2 y z+3 b y z^{3}+4 c z^{3}+R_{z}=0 ; \\
S_{1,1}(f): F(x, y, z)=0, \\
G(x, y, z)=2 y+6 b y z+12 c z^{2}+R_{z z}=0 .
\end{gathered}
$$

Since $f$ is generic, we have $c \neq 0$.

Set

$$
\begin{aligned}
x^{\prime} & =\frac{-\varepsilon}{\sqrt[4]{|c|}} x, y^{\prime}=\frac{-\varepsilon}{\sqrt{|c|}} y, z^{\prime}=\sqrt[4]{|c|} z, \\
X^{\prime} & =\frac{-\varepsilon}{\sqrt[4]{|c|}} X, Y^{\prime}=\frac{-\varepsilon}{\sqrt{|c|}} Y, Z^{\prime}=-\varepsilon Z,
\end{aligned}
$$

where $\varepsilon=\operatorname{Sgn} c$. Then we have, dropping primes,

$$
X=x, Y=y, Z=x z+y z^{2}+b y z^{3}-z^{4}+R, \text { ord } R \geqq 5 .
$$

Set $z^{\prime}=z+\frac{b}{4} y$ then we have, dropping primes,

$$
X=x, Y=y, Z=x z+y z^{2}-z^{4}+R, \text { ord } R \geqq 5 .
$$

3. Deformation of the singularities. We shall consider in this section deformations of the singularities. First of all we consider the elimination of the cusp points of generic mapping ${ }^{4)}$ of $R^{2}$ to $R^{2}$.

Lemma 1. Let $f$ be a mapping of $R^{2}$ to $R^{2}$ represented by

$$
X=x, Y=\rho(x) y-y^{3},
$$

where $\rho( \pm a)=0, \rho(x)<0$ for $|x|<a$ and $\left.\frac{d \rho}{d x}\right|_{ \pm a} \neq 0$. Then the singularities $S_{1,1}(f)$ are two points $( \pm a, 0)$ which are the cusp points of $f$; We may takes, in a neighborhood $U$ of the $C=\{(x, 0) ;|x| \leqq a\}$, a good 0-approximation $\bar{f}$ of $f$ such that $S_{1,1}(\bar{f})=\varnothing$.

Proof. Put $\varepsilon^{\prime}=2 \operatorname{Max}_{a \leqq|x| \leqq a+8} \rho(x)$. We may take smooth functions $\nu(x)$ and $\eta(x)$ which have the following properties:

$$
\begin{aligned}
& \nu(x) \geqq 0, \nu(x)=0 \text { for }|x| \geqq a+\varepsilon, \nu(x)>-\rho(x), \\
& \eta(x)=0 \text { for }|x| \geqq a+\varepsilon, \eta(x)>0 \text { for }|x|<a+\varepsilon, \\
& \frac{\nu(x)}{\eta(x)}<\frac{\varepsilon^{\prime 2}}{4}, \rho(x) \cdot \eta(x)<\nu(x) \text { for } a \leqq|x| \leqq a+\varepsilon .
\end{aligned}
$$

4) In [4] the generic mapping is refered to as the excellent mapping. 
Put

$$
\alpha(x, y)=-\eta(x) y^{3}+\nu(x) y,
$$

and take a smooth function $\beta(x, y)$ with the following properties;

$$
\begin{aligned}
& \beta(x, y)=\alpha(x, y) \text { for }|y|<\frac{9}{10} \sqrt{\frac{\nu(x)}{\eta(x)}}, \\
& \beta(x, y)=0 \quad \text { for }|y| \geqq \sqrt{\frac{\nu(x)}{\eta(x)}}, \\
& \beta(x, y) \text { and } \frac{\partial \beta}{\partial y} \text { are monotone for } \frac{9}{10} \sqrt{\frac{\nu(x)}{\eta(x)}} \leqq|y| \leqq \sqrt{\frac{\nu(x)}{\eta(x)}} .
\end{aligned}
$$

Let $f^{\prime}: R^{2} \rightarrow R^{2}$ be a mapping represented by

$$
X=x, Y=\rho(x) y-y^{3}+\beta(x, y) .
$$

Then $f^{\prime}$ is a good 0 -approximation of $f$ in the neighborhood of $C$. The singularity $S_{1}\left(f^{\prime}\right)$ is represented by

$$
F(x, y)=\rho(x)-3 y^{2}+\frac{\partial \beta}{\partial y}=0 .
$$

In the consideration of $S_{1,1}\left(f^{\prime}\right)$ we may suppose that $|x| \leqq a+\varepsilon$.

Case 1): $a \leqq|x| \leqq a+\varepsilon$. The functions $\left(\rho(x)-3 y^{2}\right)$ and $\frac{\partial \beta}{\partial y}$ are monotone for $0 \leqq|y| \leqq \sqrt{\frac{\nu(x)}{3 \eta(x)}}$, and $F(x, 0)=\rho(0)+\nu(0)>0$. On the other hand, it follows from (3) that $\frac{\partial \beta}{\partial y}$ and $\left(\rho(x)-3 y^{2}\right)$ are negative for $|y| \geqq \sqrt{\frac{\nu(x)}{3 \eta(x)}}\left(>\sqrt{\frac{\rho(x)}{3}}\right)$. Hence $F(x, y)=0$ has only tow solutions for a fixed $x$.

Case 2): $|x| \leqq a . \quad$ In this case, if $|y|<\sqrt{\frac{\nu(x)}{3 \eta(x)}}$ then $\left(\rho(x)-3 y^{2}\right)$ and $\frac{\partial \beta}{\partial y}$ are negative.

We have

$$
F(x, y)=-3(1+\eta(x)) y^{2}+(\rho(x)+\nu(x)) \text { for }|y| \leqq \sqrt{\frac{\nu(x)}{3 \eta(x)}},
$$

and $1+\eta(x)>0$ ane $\rho(x)+\nu(x)>0$. Hence $F(x, y)$ has only two solutions in $U$ for $|x| \leqq a+\varepsilon$. From these results we conclude that $S_{1,1}\left(f^{\prime}\right)$ is empty. 
Given a generic mapping $f$ of $M^{3}$ to $R^{3}$ and a point $p$ of of $S_{1,1,1}(f)$, we shall define the index at $p$ as follows.

Definition of the index. Take a fixed orientation of $M^{3}$, and let $p$ be a point of $S_{1,1,1}(f)$, Then $f$ is represented in suitable coordinate systems at $p$ and at $f(p)$ as follows:

$$
X=x, Y=y, Z=x z+y z^{2}-z^{4}+R, \text { ord } R \geqq 5 .
$$

Let $x(p), y(p)$ and $z(p)$ be the tangent vectors of $x-, y$ - and $z$-axis at $p$ whose orientations are given by the direction of coordinate axes. Consider the oriented frame $\{x(p), y(p), z(p)\}$. Then we define that the index of $p$ is +1 or -1 according as the orientation of the frame coincide with that of $M^{3}$ or not.

Lemma 2. The above definition of the index does not depend on the choice of coordinate systems.

Proof. Take two pairs of coordinate systems $\{(x, y, z),(X$, $Y, Z)\}$ and $\{(\tilde{x}, \tilde{y}, \tilde{z}),(\tilde{X}, \tilde{Y}, \tilde{Z})\}$. We may suppose that $f$ is represented in these coordinate systems as follows:

$$
\begin{aligned}
& X=x, Y=y, Z=x z+y z^{2}-z^{4}+R, \text { ord } R \geqq 5, \\
& \tilde{X}=\tilde{x}, \tilde{Y}=\tilde{y}, \tilde{Z}=\tilde{x} \tilde{z}+\tilde{y} \tilde{z}^{2}-\tilde{z}^{4}+\widetilde{R}, \text { ord } \widetilde{R} \geqq 5 .
\end{aligned}
$$

Let

$$
\begin{aligned}
& \tilde{x}=\rho(x, y, z), \tilde{y}=\psi(x, y, z), \tilde{z}=\rho(x, y, z), \\
& \tilde{X}=\Phi(X, Y, Z), \tilde{Y}=\Psi(X, Y, Z), \tilde{Z}=P(X, Y, Z) .
\end{aligned}
$$

Then the following relations hold:

$$
\begin{aligned}
& \Phi\left(x, y, x z+y z^{2}-z^{4}+R\right)=\mathcal{P}(x, y, z) \\
& \Psi\left(x, y, x z+y z^{2}-z^{4}+R\right)=\psi(x, y, z) \\
& \mathrm{P}\left(x, y, x z+y z^{2}-z^{4}+R\right)= \\
& \quad(x, y, z) \cdot \rho(x, y, z)-\psi(x, y, z) \cdot(\rho(x, y, z))^{2} \\
& \quad-(\rho(x, y, z))^{4}+\widetilde{R}(\mathcal{P}, \psi, \rho) .
\end{aligned}
$$

Put $\left.\mathcal{P}_{i j k}\right|_{0}=\frac{\partial^{i+j+k}}{\partial x^{i} \partial y^{j} \partial z^{k}} \varphi(0,0,0)$ and $J=x+2 y z-4 z^{3}+R_{001}$.

By applying $\left.\frac{\partial}{\partial z}\right|_{0}$ to $(6)$ and (7), it follows that $\left.\mathcal{P}_{00 i}\right|_{0}=\left.\mathcal{P}_{00 i}\right|_{0}=0$ 
for $i \leqq 3$. Since $(\mathcal{\rho}, \psi, \rho)$ is a coordinate transformation on $R^{3}$ we have

$$
\left.\rho_{001}\right|_{0} \neq 0
$$

By applying to $\left.\frac{\partial^{2}}{\partial y \partial z}\right|_{0}$ (8), we have

$$
\left(\left.\mathcal{P}_{010}\right|_{0}\right) \cdot\left(\left.\rho_{001}\right|_{0}\right)=0,\left.\mathcal{P}_{010}\right|_{0}=0,\left.\psi_{010}\right|_{0} \neq 0 \text {. }
$$

Furthermore applying of $\left.\frac{\partial^{3}}{\partial y \partial z^{2}}\right|_{0}$ and $\left.\frac{\partial^{4}}{\partial z^{4}}\right|_{0}$ to (8) imply

$$
\left.\mathrm{P}_{001}\right|_{0}=\left(\left.\psi_{010}\right|_{0}\right) \cdot\left(\left.\rho_{001}\right|_{0}\right)^{2}
$$

and

$$
\left.\mathrm{P}_{001}\right|_{0}=\left(\left.\rho_{001}\right|_{0}\right)^{4} .
$$

From (9), (10) and (11) we have

$$
\left.\psi_{010}\right|_{0}>0 \text {. }
$$

Applying of $\frac{\partial^{2}}{\partial x \partial z}$ to (8) implies

$$
\left.\mathrm{P}_{001}\right|_{0}=\left(\left.\mathcal{P}_{100}\right|_{0}\right) \cdot\left(\left.\rho_{001}\right|_{0}\right) .
$$

Hence we have

$$
\left(\left.\mathcal{P}_{100}\right|_{0}\right) \cdot\left(\left.\rho_{001}\right|_{0}\right)=\left(\left.\rho_{001}\right|_{0}\right)^{4}>0 .
$$

Consequently the Jacobian of the transformation $(\mathcal{P}, \psi, \rho)$ is positive at $p$.

Definition of the positive and negative sides at a point of $S_{1,1,1}$.

Let $p \in S_{1,1,1}(f)$ be a point, and consider the tangent plane $T_{S_{1}(f)}(p)$ of $S_{1}(f)$ at $p$ and the null space $N(p)$ at $p$. Then the side in $T_{S_{1}(f)}(p)$ with respect to $N(p)$ which contains the tangent vector $y(p)$ of the $y$-axis is called positive. The another side is called negative.

The inequality (12) justifies this definition.

Let $p$ be a point of $S_{1,1}(f)-S_{1,1,1}(f)$. Then, in a neighborhood of $p$, the mapping $f$ is represented in suitable coordinate systems as follows :

$$
X=x, Y=y, Z=y z-z^{3}+R, \text { ord } R \geqq 4 .
$$


Lemma 3. Let $T_{S_{1}(f)}(p)$ be the tangent plane of $S_{1}(f)$ at $p$. The tangent vector $y(p)$ of the $y$-axis is transversal to $T_{S_{1}(f)}(p)$. For any choice of coordinate systems, vestoy $y(p)$ dirests the same side with respect to $T_{S_{1}(f)}(p)$.

Proof. Suppose that $f$ is represented in another coordinate systems as follows :

$$
\tilde{X}=\tilde{x}, \tilde{Y}=\tilde{y}, \tilde{Z}=\tilde{y} \tilde{z}-\tilde{z}^{3}+\tilde{R}, \text { ord } \tilde{R} \geqq 4 .
$$

Then the similar method in the last lemma proves

$$
\left.\frac{\partial \tilde{y}}{\partial x}\right|_{0}=\left.\frac{\partial \tilde{y}}{\partial z}\right|_{0}=0,\left.\frac{\partial \tilde{y}}{\partial y}\right|_{0}>0,\left.\frac{\partial \tilde{z}}{\partial x}\right|_{0}=0 .
$$

Definition of the positive and negative sides at a point of $S_{1,1}$.

Let $p \in S_{1,1}(f)$ be point, and consider the tangent space $T_{M^{3}}(p)$ of $M^{3}$ at $p$ and the tangent plane $T_{S_{1}(f)}(p)$ of $S_{1}(f)$ at $p$. Then the side in $T_{M^{3}}(p)$ with respect to $T_{S_{1}(f)}(p)$ which contains the tangent vector $y(p)$ of the $y$-axis is called positive. The another side is called negative.

This definition is justified by Lemma 3 .

Theorem 1. Let $f$ be a generic mapping of $M^{3}$ to $R^{3}$, and let $p$ and $q$ be points of $S_{1,1,1}(f)$. Suppose that the following conditions:

1) $p$ and $q$ are in the same connected component of $S_{1}(f)$.

2) There is a smooth simple curve $C$ in $S_{1}(f)$ which starts from $p$ into the negative side, ends at $q$ from the negative side and does not touch any other singularities $S_{1,1}(f)$.

3) The indices of $p$ and $q$ are different.

Then we may take, in a tubular neighborhood $U(C)$ of $C$, a good 2-approximation $\bar{f}$ of $f$ such that $S_{1,1,1}(\bar{f}) \cap U(C)=\varnothing$.

Before proving this theorem we prepare the following lemmas.

Lemma 4. Under the same conditions as in the last theorem, we may choose a coordinate system $(x, y, z)$ in $U(C)$ and a parameter system $(X, Y, Z)$ in $f(U(C))$ in which $C$ is represented as the set $\{(0, y, 0) ;|y| \leqq 1\}$, and $f$ is represented as follows :

$$
X=x, Y=y, Z=x z+\rho(y) z^{2}-z^{4}+R, \operatorname{ord}_{z} R \geqq 5
$$


with smooth function $\rho(y)$ satisfying $\rho( \pm 1)=0, \rho(y)<0$ for $|y|<1$, $\frac{d \rho}{d y}(-1)<0$ and $\frac{d \rho}{d y}(+1)>0$.

Proof. Take a Riemannian metric in $M^{3}$, and consider a smooth open curve $C^{\prime} \supset C$. Let $\varepsilon$ be a sufficient small positive number. Parametrize $C^{\prime}$ by $(-1-\varepsilon, 1+\varepsilon)$ and $C$ by $[-1,1]$. Take a smooth vector field $\left\{V_{p}\right\}$ on $C^{\prime}$ such that each vector $V_{p}$ is transversal to the tangent vector of $C^{\prime}$ and the null space $N(p)$ at $p$. For each point $p \in C^{\prime}$, consider the geodesic $g_{p}$ whose tangent vector at $p$ is $V_{p}$. Let $D_{p}$ be the set of points $q$ of $g_{p}$ such that the length of the geodesic between $p$ and $q$ is less than $\varepsilon$, and put $D=\bigcup_{p \in C^{\prime}} D_{p}$. Then, for sufficient small $\varepsilon, D$ is an open 2-disk which contains $C$, and the mapping $f \mid D$ is a local homeomorphism. Let $L_{f(q)}$ denote the line segment which is normal to $f(D)$ at $f(q)$. Then it follows that $M_{q}=\left\{f^{-1}\left(L_{f(q)}\right) ; q \in D\right\}$ is a family of curves and that the set of points $r \in M_{q}(q \in D)$ is a tubular neighborhood of $C$ [2]. In virtue of the above definitions of $C^{\prime}, D_{p}, L_{f(q)}$ and $M_{q}$, we may take the following coordinate system $(x, y, z)$ in a small tubular neighborhood $U(C)$ of $C$ and the following parameter system $(X, Y, Z)$ in $f(U(C))$. Let $r$ be a point of $U(C)$, then $r$ is a point of $M_{q}, q \in D$, and $q$ is a point of $g_{p}, p \in C^{\prime}$. We take $(x, y, z)$ as coordinates of $r$ such that

i) $x$ is the length in $g_{p}$ from $p$ to $q$.

ii) $y$ is the parameter on $C^{\prime}$.

iii) $z$ is the length in $M_{q}$ from $q$ to $r$.

For the set $f(U(C))$, we may define $(X, Y, Z)$ as follows: Let $f(r)$ be a point of $f(U(C))$ and $(x, y, z)$ be the coordinate of $r$. We set

$$
X=x, Y=y \text { and } Z=\text { the length in } L_{f(q)} \text { from } f(q) \text { to } f(r) .
$$

Then the mapping $f$ is represented in the neighborhood $U(C)$ as follows:

$$
X=x, Y=y, Z=h(x, y, z) .
$$

Expanding $h$ with respect to $z$, we have 


$$
Z=\sum_{i=0}^{4} \rho_{i}(x, y) z^{i}+R, \operatorname{ord}_{z} R \geqq 5 .
$$

In virtue of the definition of the parameters $z, Z$, we have $Z=0$ if $z=0$. Hence we have $\rho_{0}(x, y)=0$. Consider now the set $S_{1}(f)$ represented by

$$
\rho_{1}(x, y)+2 \rho_{2}(x, y) z+3 \rho_{3}(x, y) z^{2}+4 \rho_{4}(x, y) z^{3}+R_{z}=0 .
$$

Since the $y$-axis is contained in $S_{1}(f)$, we have $\rho_{1}(0, y)=0$.

Hence We may set

$\rho_{1}(x, y)=\rho_{11}(y) x+\rho_{12}(x, y) x^{2}$ and $\rho_{2}(x, y)=\rho_{20}(y)+\rho_{21}(x, y) x$.

Then we have

$$
\begin{aligned}
Z= & \rho_{11}(y) x z+\rho_{12}(x, y) x^{2} z+\rho_{20}(y) z^{2}+\rho_{21}(x, y) x z^{2} \\
& +\rho_{3}(x, y) z^{3}+\rho_{4}(x, y) z^{4}+R .
\end{aligned}
$$

Since $C^{\prime} \cap S_{1,1}(f)$ are only two points $(0, \pm 1,0)$, we have

$$
\rho_{20}( \pm 1)=0 \text { and } \rho_{20}(y) \neq 0 \text { for } y \neq \pm 1 .
$$

Now the mapping $f$ is generic, therefore the expansion of $h$ must contain the term of order 2. Hence we have $\rho_{11}( \pm 1)=0$.

The equation 3) in Section 1 and 13) show that

$$
\frac{d \rho_{20}}{d y}(-1) \frac{d \rho_{20}}{d y}(+1)>0 \text {. }
$$

The condition 3) in Theorem 1 and 14) show that $\rho_{11}(-1) \rho_{11}(+1)$ $>0$.

Take a smooth function $\rho_{11}^{\prime}(y)$ such that $\rho_{11}^{\prime}(y) \neq 0$ and $\rho_{11}^{\prime}(y)$ $=\rho_{11}(y)$ for $y$ near \pm 1 , and set

$$
x^{\prime}=\rho_{11}^{\prime}(y) x, \bar{\rho}_{11}(y)=\frac{\rho_{11}(y)}{\rho_{11}^{\prime}(y)} .
$$

Then we have $\bar{\rho}_{11}(y)=1$ for $y$ near \pm 1 and, dropping primes, we have

$$
\begin{aligned}
Z= & \bar{\rho}_{11}(y) x z+\bar{\rho}_{12}(x, y) x^{2} z+\rho_{20}(y) z^{2}+\bar{\rho}_{21}(x, y) x z^{2}+\rho_{3}^{\prime}(x, y) z^{3} \\
& +\rho_{4}^{\prime}(x, y) z^{4}+R^{\prime}, \operatorname{ord}_{z} R^{\prime} \geqq 5 .
\end{aligned}
$$

Consider now a smooth function defined by 
and set

$$
\beta(y)=\frac{\bar{\rho}_{11}(y)-1}{2 \rho_{20}(y)}
$$

$$
x^{\prime}=x, y^{\prime}=y, z^{\prime}=z+\beta(y) x .
$$

We have, dropping primes,

$$
\begin{aligned}
X= & x, Y=y, Z=x z+\bar{\rho}_{2}(x, y) z^{2}+\bar{\rho}_{3}(x, y) z^{3} \\
& +\bar{\rho}_{4}(x, y) z^{4}+\bar{R}, \text { ord }_{z} \bar{R} \geqq 5,
\end{aligned}
$$

with $\bar{\rho}_{2}(0, y)=\rho_{20}(y)$.

- Set

$$
x^{\prime}=x, y^{\prime}=y, z^{\prime}=z+\bar{\rho}_{21}(x, y) z^{3}
$$

where $\bar{\rho}_{2}(x, y)=\rho_{20}(y)+\overline{\bar{\rho}}_{21}(x, y) x$.

Then we have, dropping primes,

$$
\begin{aligned}
X= & x, Y=y, Z=x z+\rho_{20}(y) z^{2}+\overline{\bar{\rho}}_{3}(x, y) z^{3} \\
& +\overline{\bar{\rho}}_{4}(x, y) z^{4}+\overline{\bar{R}}, \operatorname{ord}_{z} \overline{\bar{R}} \geqq 5 .
\end{aligned}
$$

Set

$$
x^{\prime}=x, y^{\prime}=y, z^{\prime}=z+\rho_{31}(x, y) z^{3}
$$

where $\bar{\rho}_{3}(x, y)=\rho_{30}(y)+\rho_{31}(x, y) x$.

Then we have, dropping primes,

$$
\begin{aligned}
X= & x, Y=y, Z=x z+\rho_{20}(y) z^{2}+\rho_{30}(y) z^{3} \\
& +\tilde{\rho}_{4}(x, y) z^{4}+\widetilde{R}, \operatorname{ord}_{z} \widetilde{R} \geqq 5 .
\end{aligned}
$$

Since the points $(0, \pm 1,0)$ are the points of $S_{1,1,1}(f)$, the argument in Section 1 follows that

$$
\rho_{30}( \pm 1)=0 \text {. }
$$

Hence we may define the following coordinate transformation

$$
x=x^{\prime}, y=y^{\prime}, z=z^{\prime}-\frac{\rho_{30}\left(y^{\prime}\right)}{2 \rho_{20}\left(y^{\prime}\right)} z^{\prime 2} .
$$

Then we have, dropping primes, $X=x, Y=y, Z=x z+\rho(x, y) z^{2}-\nu(x, y) z^{4}+R, \operatorname{ord}_{z} R \geqq 5$.

The function $\rho(x, y)$ satisfies the following conditions : 


$$
\begin{aligned}
& \rho(0, y)=\rho_{20}(y), \rho(0, \pm 1)=0, \\
& \rho(0, y) \neq 0 \text { for } y \neq \pm 1, \\
& \frac{\partial \rho}{\partial y}(0,-1) \cdot \frac{\partial \rho}{\partial y}(0,+1)<0 .
\end{aligned}
$$

From the above properties of $\rho$, it follows that there exist smooth functions $\varphi(x)$ and $\psi(x)$ satisfying the following conditions :

$$
\rho(x, \mathcal{P}(x))=0, \mathcal{P}(0)=-1 ; \rho(x, \psi(x))=0, \varphi(0)=1 .
$$

Set

$$
\begin{aligned}
& x=x^{\prime}, \quad y=\left(\psi\left(x^{\prime}\right)-\mathcal{P}\left(x^{\prime}\right)\right)\left(\frac{y^{\prime}+1}{2}\right)+\mathcal{P}\left(x^{\prime}\right), z=z^{\prime}, \\
& X=X^{\prime}, \quad Y=\left(\psi\left(X^{\prime}\right)-\mathcal{P}\left(X^{\prime}\right)\right)\left(\frac{Y^{\prime}+1}{2}\right)+\mathcal{P}\left(X^{\prime}\right), Z=Z^{\prime} .
\end{aligned}
$$

Then we have

$$
X^{\prime}=x^{\prime}, Y^{\prime}=y^{\prime}, Z^{\prime}=x^{\prime} z^{\prime}+\rho^{\prime}\left(x^{\prime}, y^{\prime}\right){z^{\prime}}^{2}-\nu\left(x^{\prime}, y^{\prime}\right) z^{\prime 4}+R^{\prime},
$$

$\operatorname{ord}_{z^{\prime}} R^{\prime} \geqq 5$, with $\rho^{\prime}\left(x^{\prime}, y^{\prime}\right)=\rho\left(x^{\prime},\left(\psi\left(x^{\prime}\right)-\varphi\left(x^{\prime}\right)\right)\left(\frac{y^{\prime}+1}{2}\right)+\varphi\left(x^{\prime}\right)\right)$.

The function $\rho^{\prime}$ has the following properties

$$
\rho^{\prime}\left(x^{\prime}, \pm 1\right)=0, \rho^{\prime}\left(0, y^{\prime}\right)=\rho\left(0, y^{\prime}\right) .
$$

We may now define a smooth function

$$
\sigma\left(x^{\prime}, y^{\prime}\right)=\frac{\rho^{\prime}\left(x^{\prime}, y^{\prime}\right)}{\rho^{\prime}\left(0, y^{\prime}\right)} .
$$

We have $\sigma\left(x^{\prime}, y^{\prime}\right)>0$ for $\left|x^{\prime}\right|<\varepsilon$ and $\left|y^{\prime}\right|<1+\varepsilon$.

Set

$$
\begin{aligned}
& x^{\prime}=\sqrt{\sigma\left(x^{\prime}, y^{\prime}\right)} x, y^{\prime}=y, \quad z^{\prime}=\frac{1}{\sqrt{\sigma\left(x^{\prime}, y^{\prime}\right)}} z \\
& X^{\prime}=\sqrt{\sigma\left(X^{\prime}, Y^{\prime}\right)} X, Y^{\prime}=Y, Z^{\prime}=Z .
\end{aligned}
$$

We have

$$
X=x, Y=y, Z=x z+\rho(0, y) z^{2}-\nu^{\prime \prime}(x, y) z^{4}+R, \operatorname{ord}_{z} R \geqq 5 .
$$

Take the expansion

$$
\nu^{\prime \prime}(x, y)=\nu(y)+\nu_{1}(x, y) x,
$$


and set

$$
x^{\prime}=x, y^{\prime}=y, z^{\prime}=z-\nu_{1}(x, y) z^{4} .
$$

Then we have, dropping primes,

$$
X=x, Y=y, Z=x z+\rho(y) z^{2}-\nu(y) z^{4}+R, \operatorname{ord}_{z} R \geqq 5 .
$$

where $\rho(y)=\rho(0, y)$ and $\nu(y)=\nu^{\prime \prime}(0, y)$.

Since $f$ is generic, we have $\nu( \pm 1)=0$. We may suppose that $\nu(-1)>0$. Then the condition 2) in Theorem 1 follows that

$$
\frac{d \rho}{d y}(-1)<0 \text { and } \rho(y)<0 \text { for }|y|<1 .
$$

Therefore the condition 2) in Theorem 1 and 14) show that $\nu(+1)>0$.

Now we may take a smooth function $\nu^{\prime}(y)>0$ such that $\nu^{\prime}(y)=$ $\nu(y)$ for $y$ near

Set

$$
\begin{aligned}
& x^{\prime}=\frac{1}{\sqrt[4]{\nu^{\prime}(y)}} x, y^{\prime}=y, z^{\prime}=\sqrt[4]{\nu^{\prime}(y)} z, \\
& X^{\prime}=\frac{1}{\sqrt[4]{\nu^{\prime}(Y)}} X, Y^{\prime}=Y, Z^{\prime}=Z .
\end{aligned}
$$

Then we have, dropping primes,

$$
X=x, Y=y, Z=x z+\bar{\rho}(y) z^{2}-\bar{\nu}(y) z^{4}+\bar{R}, \operatorname{ord}_{z} \bar{R} \geqq 5 .
$$

It holds that $\bar{\nu}(y)=1$ for $y$ near \pm 1 .

Set

$$
x=x^{\prime}, y=y^{\prime}, z=z^{\prime}+\frac{(\bar{\nu}(y)-1)}{2 \bar{p}(y)}{\boldsymbol{z}^{\prime}}^{3} .
$$

Then we have, dropping primes,

$$
X=x, Y=y, Z=x z+\bar{\rho}(y) z^{2}+\frac{\bar{\nu}(y)-1}{2 \bar{\rho}(y)} x z^{3}-z^{4}+R, \operatorname{ord}_{z} R \geqq 5 .
$$

Again set

$$
x=x^{\prime}, y=y^{\prime}, z=z^{\prime}-\frac{\bar{\nu}\left(y^{\prime}\right)-1}{4\left(\bar{\rho}\left(y^{\prime}\right)\right)^{2}} x^{\prime} z^{\prime 2} .
$$

Then we have, dropping primes,

$$
X=x, Y=y, Z=x z+\tilde{\rho}(x, y) z^{2}-\widetilde{\nu}(x, y) z^{4}+\widetilde{R}, \operatorname{ord}_{z} \widetilde{R} \geqq 5 ;
$$


$\tilde{\rho}(x, y)$ has the same properties as in 16$)$ and $\tilde{\nu}(0, y)=1$ for $|y|$ $<1+\varepsilon$.

Hence, by repeating the method in the preceding part, we may represent $f$ as follows :

$$
X=x, \quad Y=y, \quad Z=x z+\rho(y) z^{2}-z^{4}+R, \quad \operatorname{ord}_{z} R \geqq 5 .
$$

The following lemma is easily proved by Lemma 4 .

Lemma 5. In the same conditions as in the theorem 1, we may take, in a neighborhood $U$ of $C$, a good 4-approximation $\bar{f}$ of $f$ which is represented by the following equations:

$$
X=x, \quad Y=y, \quad Z=x z+\rho(y) z^{2}-z^{4},
$$

with smooth function $\rho(y)$ satisfying $\rho( \pm 1)=0, \frac{d \rho}{d y}(-1)<0, \frac{d \rho}{d y}(+1)$ $>0, \rho(y)<0$ for $|y|<1$.

Proof of Theorem 1. By Lemma 5, we may suppose that $f$ is represented as follows :

$$
X=x, \quad Y=y, \quad Z=x z+\rho(y) z^{2}-z^{4}
$$

where $|y|<1+\varepsilon,|x|<\varepsilon,|z|<\varepsilon, \rho( \pm 1)=0, \frac{d \rho}{d y}(-1)<0, \frac{d \rho}{d y}(+1)$ $>0$ and $\rho(y)<0$ for $|y|<1$.

Let $\varepsilon^{\prime}>0$ be a positive number such that $\frac{\sqrt{\varepsilon^{\prime}}}{2}<\varepsilon$. Then we may take a positive number $\varepsilon^{\prime \prime}$ such that $\varepsilon^{\prime \prime}<\varepsilon$ and $\underset{1 \leqq|y| \leqq 1+\varepsilon^{\prime \prime}}{2}$ $\rho(y)<\varepsilon^{\prime}$.

We may now take a smooth function $\nu(y)$ which has the following properties :

$$
\begin{aligned}
& \nu(y)>0 \text { for }|y|<1+\varepsilon^{\prime \prime}, \quad \nu(y)=0 \text { for }|y| \geqq 1+\varepsilon^{\prime \prime}, \\
& \nu(y)>-\rho(y) .
\end{aligned}
$$

Put

$$
\eta_{0}(z)=\frac{2}{\varepsilon^{\prime}} z^{4}-z^{2}+\frac{\varepsilon^{\prime}}{8}
$$

and take a smooth function $\eta(z)$ satisfying the following properties : 
i) $\eta(z)=\eta_{0}(z)$ for $|z| \leqq \frac{9}{20} \sqrt{\varepsilon^{\prime}}, \eta(z)=0$ for $|z| \geqq \frac{\sqrt{\varepsilon^{\prime}}}{2}$,
ii) $\eta(z), \frac{d \eta}{d z}$ are monotone for $\frac{9}{20} \sqrt{\varepsilon^{\prime}}<|z|<\frac{\sqrt{\varepsilon^{\prime}}}{2}$.

We may then take a positive number $\varepsilon^{\prime \prime \prime}$ which has the following properties.

1) If $|x|>\varepsilon^{\prime \prime \prime}$, we have $\left|x+2 \rho(y) z-4 z^{3}\right|>\frac{4}{3} \nu(y) \sqrt{\frac{\varepsilon^{\prime}}{12}}$ for $|y| \leqq 1+\varepsilon,|z| \leqq \frac{\sqrt{\varepsilon^{\prime}}}{2}$.

2) $\varepsilon^{\prime \prime \prime}>2 \nu(y) \sqrt{\frac{\varepsilon^{\prime}}{12}}$ for $|y| \leqq 1+\varepsilon$.

$\varepsilon^{\prime \prime \prime}$ is sufficiently small if so is $\varepsilon^{\prime}$. Hence we may suppose that $2 \varepsilon^{\prime \prime \prime}<\varepsilon$. For such $\varepsilon^{\prime \prime \prime}$, we may take a smooth function $\phi(x)$ satisfying the following properties :

$\varphi(x)=\varphi(-x) \geqq 0, \varphi(x)=1$ for $|x| \leqq \varepsilon^{\prime \prime \prime}, \phi(x)=0$ for $|x| \geqq 3 \varepsilon^{\prime \prime \prime}$, and $\left|\frac{d \Phi}{d x}\right|<\frac{1}{\varepsilon^{\prime \prime \prime}}$.

Now we may define a mapping $\bar{f}$ by the following equation:

$$
X=x, . Y=y, \quad Z=x z+\rho(y) z^{2}-z^{4}-\varphi(x) \nu(y) \eta(z) .
$$

Then we have $f(x, y, z)=\bar{f}(x, y, z)$ for $|x| \geqq 3 \varepsilon^{\prime \prime \prime}$ or $|y| \geqq 1+\varepsilon^{\prime \prime}$ or $|z| \geqq \frac{\sqrt{\varepsilon^{\prime}}}{2}$.

Hence the mapping $\bar{f}$ is a good 1-approximation of $f$. We shall next consider the singularities of $\bar{f}$. In this case we may suppose that $|x| \leqq 3 \varepsilon^{\prime \prime \prime},|y| \leqq 1+\varepsilon^{\prime \prime}$ and $|z| \leqq \frac{\sqrt{\varepsilon^{\prime}}}{2}$. The set $S_{1}(\bar{f})$ is represented by

$$
F(x, y, z)=x+2 \rho(y) z-4 z^{3}+\mathcal{P}(x) \nu(y) \frac{d \eta}{d z}(z)=0 .
$$

We have

$$
F_{x}=1-\frac{d \rho}{d x} \nu(y) \frac{d \eta}{d z} \text { and }\left|\frac{d \rho}{d x} \nu(y) \frac{d \eta}{d z}\right|<\frac{\nu(y)}{\varepsilon^{\prime \prime \prime}} \frac{4}{3} \sqrt{\frac{\varepsilon^{\prime}}{12}}<1 .
$$

Hence the set $S_{1}(\bar{f})$ is a regular submanifold and the set $S_{1,1}(\bar{f})$ is represented by

$$
F(x, y, z)=0, \quad G(x, y, z)=2 \rho(y)-12 z^{2}-\mathcal{P}(x) \nu(y) \frac{d^{2} \eta}{d z^{2}}=0 .
$$


If $|x|>\varepsilon^{\prime \prime \prime}$, then we have $F \neq 0$. If $|z| \geqq \sqrt{\frac{\varepsilon^{\prime}}{12}}$, then we have $G<0$ because of $\frac{d^{2} \eta}{d z^{2}}>0$ and $\rho(y)<\frac{\varepsilon^{\prime}}{2}$. Therefore we may suppose that $|x| \leqq \varepsilon^{\prime \prime \prime}$ and $|z|<\sqrt{\frac{\varepsilon^{\prime}}{12}}$. In this case, the set $S_{1,1}(\bar{f})$ is represented by

$$
\begin{gathered}
x+2(\rho(y)+\nu(y)) z-4\left(1+\frac{2 \nu(y)}{\varepsilon^{\prime}}\right) z^{2}=0, \\
(\rho(y)+\nu(y))-6\left(1+\frac{2 \nu(y)}{\varepsilon^{\prime}}\right) z^{2}=0 .
\end{gathered}
$$

Hence, for a fixed $y, S_{1,1}(\bar{f})$ in $U$ consists of only two points :

$$
\left(\mp \frac{5}{3}(\nu(y)+\rho(y)) \sqrt{\frac{\varepsilon^{\prime}(\nu(y)+\rho(y))}{6\left(2 \nu(y)+\varepsilon^{\prime}\right)}}, \quad y, \pm \sqrt{\left.\frac{\varepsilon^{\prime}(\nu(y)+\rho(y))}{6\left(2 \nu(y)+\varepsilon^{\prime}\right)}\right)} .\right.
$$

Moreover we consider $G_{z}(x, y, z)$ for points of $S_{1,1}(\bar{f})$ in $U$. Then we have $G_{z}(x, y, z)=-24\left(1+\frac{2 \nu(y)}{\varepsilon^{\prime}}\right) z$ because of $|x|<\varepsilon^{\prime \prime \prime}$ and $|z|<\sqrt{\frac{\varepsilon^{\prime}}{12}}$. Hence we have $G_{z}(x, y, z)=1=0$ for points of $S_{1,1}(\bar{f})$ in $U$. Since $S_{1,1,1}(\bar{f})$ is represented by $F=G=G_{z}=0$, $S_{1,1,1}(\bar{f}) \cap U=\varnothing$.

Lemma 6. Let $C$ be a circle or a simple arc in $S_{1,1}(f)$. Suppose that $C$ contains no point of $S_{1,1,1}(f)$. Then the mapping $f$ is represented in a neighborhood of $C$ as follows:

$$
X=x, \quad Y=y, \quad Z=y z-z^{3}+R, \quad \operatorname{ord}_{z} R \geqq 4,
$$

where $C$ is represented by $y=z=0$, and $x, X$ are real numbers mod 1 or real numbers in $[0,1]$ according as $C$ is a circle or a simple arc.

Proof. Consider a Riemannian metric tn $M^{3}$. Then we may take a vector field $\left\{V_{p}\right\}$ on $C$ such that each $V_{p}$ is the normal vector of $S_{1}(f)$ at $p$. For each $p \in C$, consider the geodesic $g_{p}$ whose tangent vector at $p$ is $V_{p}$. Let $D_{p}$ be the set of points $q$ of the geodesic $g_{p}$ such that the length of the geodesic between $p$ and $q$ is less than $\varepsilon$ and put $D=\bigcup_{p \in C} D_{p}$. Then, for small $\varepsilon^{\prime}, D$ 
is homeomorphic to $C \times I$ where $I$ is a interval. Now the mapping $f \mid D$ is a local homeomorphism. Let $L_{f(q)}$ denote the line segment which is normal to $f(D)$ at $f(q)$. Then $\left\{M_{q}=f^{-1}\left(L_{f(q)}\right) ; q \in D\right\}$ is a family of smooth curves. Consequence, as in the proof of Lemma 4, we may take paramenter systems $(x, y, z)$ of $U(C)$ and $(X, Y, Z)$ of $f(U(C))$ in which $f$ is represented by

$$
X=x, \quad Y=y, \quad Z=h(x, y, z) .
$$

Expand $h$ with respect to $z$ :

$$
h(x, y, z)=\sum_{i=0}^{3} a_{i}(x, y) z^{i}+R, \quad \operatorname{ord}_{z} R \geqq 4 .
$$

Then, in the above choice of parameters, we have $a_{0}(x, y)=0$. The set $S_{1}(f)$ is represented by

$$
a_{1}(x, y)+2 a_{2}(x, y)+3 a_{3}(x, y) z^{2}+R_{z}=0 .
$$

Since $C$ is contained in $S_{1}(f)$, we have $a_{1}(x, y)=0$. Hence we may put $a_{1}(x, y)=a_{11}(x, y) y$. We have $a_{11}(x, 0) \neq 0$ since $f$ is generic.

Set

$$
x^{\prime}=x, y^{\prime}=a_{11}(x, y) y, z^{\prime}=z, \quad X^{\prime}=X, Y^{\prime}=a_{11}(X, Y) Y, Z^{\prime}=Z .
$$

Then we have, dropping primes,

$$
X=x, \quad Y=y, \quad Z=y z+a_{2}(x, y) z^{2}+a_{3}(x, y) z^{3}+R .
$$

Now $S_{1,1}(f)$ is represented by the following equations:

$$
\begin{gathered}
y+2 a_{2}(x, y) z+3 a_{3}(x, y) z^{2}+R_{z}=0, \\
2 a_{2}(x, y)+6 a_{3}(x, y) z+R_{z z}=0 .
\end{gathered}
$$

Since $C$ is contained in $S_{1,1}(f)$, we have $a_{2}(x, 0)=0$. Hence we may put $a_{2}(x, y)=a_{21}(x, y) y$.

Set

$$
x^{\prime}=x, \quad y^{\prime}=y, \quad Z^{\prime}=z+a_{21}(x, y) z^{2} .
$$

Then we have, dropping primes,

$$
X=x, \quad Y=y, \quad Z=y z+a_{3}(x, y) z^{3}+R, \quad \operatorname{ord}_{z} R \geqq 4 .
$$

Since $f$ is generic, we have $a_{3}(x, 0) \neq 0$. 
Set

$$
\begin{gathered}
x^{\prime}=x, \quad y^{\prime}=\frac{-y}{\sqrt[3]{a_{3}(x, y)}}, \quad z^{\prime}=-\sqrt[3]{a_{3}(x, y)} z, \\
X^{\prime}=X, \quad Y^{\prime}=\frac{-Y}{\sqrt[3]{a_{3}(x Y)}}, \quad Z^{\prime}=Z .
\end{gathered}
$$

Then we have, dropping primes,

$$
X=x, \quad Y=y, \quad Z=y z-z^{3}+R, \quad \operatorname{ord}_{z} R \geqq 4 .
$$

Then following lemma is easily obtained from the last lemma.

Lemma 7. Suppose that $C$ is a circle or a simple arc in $S_{1,1}(f)$ and that $C \cap S_{1,1,1}(f)=\varnothing$. Then the mapping $f$ has, in a tubular neighborhood of $C$, a good 3-approximation $\bar{f}$ represented by

$$
X=x, \quad Y=y, \quad Z=y z-z^{3} .
$$

Lemma 8. Let $p$ be a point of $S_{1,1}(f)-S_{1,1,1}(f)$, then we may take, in a neighborhood $U$ of $p$, a good 2-approximation $\bar{f}$ of $f$ such that $S_{1,1}(\bar{f}) \cap U$ is a simple curve and contains two points $q, q^{\prime}$ of $S_{1,1,1}(\bar{f})$. The indices of $q$ and $q^{\prime}$ are the same, and the positive sides of $q$ and $q^{\prime}$ are oposite with respect to $S_{1,1}(\bar{f})$. It is possible to take the indices of $q$ and $q^{\prime}$ as positive or negative.

Proof. By the last lemma, we may suppose that $f$ is represented in a neighborhood $U$ as follows:

$$
X=x, \quad Y=y, \quad Z=y z-z^{3} .
$$

Consider a function $\alpha(z)=\frac{-1}{\varepsilon^{2}} z^{5}+2 z^{3}-\varepsilon^{2} z$ for sufficiently small $\varepsilon>0$. We may then take a smooth function $\beta(z)$ satisfying:

i) $\beta(z)=\alpha(z)$ for $|z| \leqq \frac{9}{10} \varepsilon$,

ii) $\beta(z)=0$ for $|z| \geqq \varepsilon$,

iii) $\beta(z), \beta^{\prime}(z)=\frac{d \beta}{d z}$ are monotone for $\frac{9}{10} \varepsilon \leqq|z| \leqq \varepsilon$.

Take a smooth function $\sigma(x)$ such that

$$
\begin{aligned}
& \sigma(x)=\sigma(-x), \quad 0 \leqq \sigma(x) \leqq 1 \\
& \sigma(x)=1 \text { for }|x| \leqq \frac{\varepsilon}{4}, \quad \sigma(x)=0 \quad \text { for } \quad|x| \geqq \frac{\varepsilon}{2} .
\end{aligned}
$$


Consider now the mapping $\bar{f}$ represented by

$$
X=x, \quad Y=y, \quad Z=y z-z^{3}+\sigma(x) \sigma(y) \beta(z+x) .
$$

Then we have $\sigma(x) \sigma(y) \beta(z+x)=0$ for $(x, y, z) \notin U$ where $U=\{(x, y, z) ;|x| \leqq \varepsilon,|y| \leqq \varepsilon,|z| \leqq 2 \varepsilon\}$. Therefore $\bar{f}$ is a good 2-approximation of $f$ in the neighborhood $U$ of $y$. Now we shall consider the singularities of $\bar{f}$ in $U . S_{1}(\bar{f})$ is represented by the following equation

$$
F(x, y, z)=y-3 z^{2}+\sigma(x) \sigma(y) \beta^{\prime}(z+x)=0 .
$$

Since $\left|\beta^{\prime}(z+x)\right| \leqq \varepsilon^{2}$, we have $|F(. x, y, z)|>0$ for $|y|>\frac{\varepsilon}{4}$. Hence we may suppose that $|y| \leqq \frac{\varepsilon}{4}$ and the equation of $S_{1}(\bar{f})$ is

$$
y-3 z^{2}+\sigma(x) \beta^{\prime}(z+z)=0 .
$$

Thus the set $S_{1}(\bar{f})$ is siotopic in $U$ to $S_{1}(f)$. Consider $S_{1,1}(\bar{f})$ which is represented by the following equations:

$$
\begin{aligned}
& y-3 z^{2}+\sigma(x) \beta^{\prime}(z+x)=0, \\
& -6 z+\sigma(x) \beta^{\prime \prime}(z+x)=0 .
\end{aligned}
$$

Set $z^{\prime}=z+x$ and $g\left(x, z^{\prime}\right)=-6\left(z^{\prime}-x\right)+\sigma(x) \beta^{\prime \prime}\left(z^{\prime}\right)$.

Case 1: $\frac{\varepsilon}{4}<x<\frac{\varepsilon}{2}$. We have $\beta^{\prime \prime}\left(z^{\prime}\right) \geqq 0$ for $z^{\prime} \leqq-\sqrt{\frac{3}{5}} \varepsilon$. Hence we have $g\left(x, z^{\prime}\right)>0$. For $-\sqrt{\frac{3}{5}} \varepsilon<z^{\prime} \leqq 0$, we have $g\left(x, z^{\prime}\right)$ $=-6\left(z^{\prime}-x\right)+\sigma(x)\left(\frac{-20}{\varepsilon^{2}}{z^{\prime}}^{3}+12 z^{\prime}\right) \geqq \frac{6}{4} \varepsilon-\frac{4}{10} \varepsilon>0$. For $\sqrt{\frac{3}{5}} \varepsilon \leqq z^{\prime}$, we have $g\left(x, z^{\prime}\right)<0$ because $z^{\prime}-x>0$ and $\beta^{\prime \prime}\left(z^{\prime}\right) \leqq 0$. For $0<z^{\prime}$ $\leqq \sqrt{\frac{3}{5}} \varepsilon$, we have

$$
g\left(x, z^{\prime}\right)=6(2 \sigma(x)-1) z^{\prime}-\frac{2 \sigma(x)}{\varepsilon^{2}}{\boldsymbol{z}^{\prime}}^{3}+6 x .
$$

It $2 \sigma(x)-1 \leqq 0$, then $g\left(x, z^{\prime}\right)$ is monotone decreasing, $g(x, 0)>0$ and $g(x) \sqrt{\frac{3}{5}} \varepsilon<0$.

If $2 \sigma(x)-1>0$, then $g\left(x, z^{\prime}\right)>0$ for $z^{\prime}=\sqrt{\frac{2 \sigma(x)-1}{10 \sigma(x)}} \varepsilon$. 
Hence $g\left(x, z^{\prime}\right)=0$ has only one solution for $x>\frac{\varepsilon}{4}$.

Case 2: $-\frac{\varepsilon}{2}<x<-\frac{\varepsilon}{4}$. By the same argument in case 1 , we have that $g\left(x, z^{\prime}\right)=0$ has only one solution for $x<-\frac{\varepsilon}{4}$.

Thus the $\operatorname{set} S_{1,1}(\bar{f})$, for $\frac{\varepsilon}{4}<|x|$, is a simple curve.

Case 3: $|x|<\frac{\varepsilon}{4}$. The set $S_{1,1}(f)$ is represented by

$$
\begin{aligned}
& y=3 z^{2}-\beta^{\prime}(z+x), \\
& -6 z+\beta^{\prime \prime}(z+x)=0 .
\end{aligned}
$$

If $|z+x| \geqq \frac{9}{10} \varepsilon$, we have $(z+x) z>0$. By the definition of $\beta$, we have $\beta^{\prime \prime}(z+x) \cdot(z+x)<0$. Hence we have $-6 z+\beta^{\prime \prime}(z+x) \neq 0$.

If $|z+x|<\frac{9}{10} \varepsilon$, the set $S_{1,1}(\bar{f})$ is represented by

$$
\begin{gathered}
y=3 z^{2}+\frac{5}{\varepsilon^{2}}(z+x)^{4}-6(z+x)^{2}+\varepsilon^{2}, \\
3 z+6 x-\frac{10}{\varepsilon^{2}}(z+x)^{3}=0 .
\end{gathered}
$$

Hence the set $S_{1,1,1}(\bar{f})$ is represented by the above equations together with

$$
1-\frac{10}{\varepsilon^{2}}(z+x)^{2}=0
$$

Thus $S_{1,1,1}(\bar{f})$ consists of two points $\left\{q, q^{\prime}\right\}=\left\{\left( \pm \frac{2 \varepsilon}{3 \sqrt{10}}, \frac{77}{60} \varepsilon^{2}\right.\right.$, $\left.\left.\pm \frac{5 \varepsilon}{3 \sqrt{10}}\right)\right\}$. This proves the first part.

Expanding $y z-z^{3}+\beta(x+z)$ at $q$ or $q^{\prime}$, we have

$$
\begin{aligned}
Z^{\prime}= & a+\mathcal{P}\left(x^{\prime}, y^{\prime}\right)+\left(y^{\prime}+\psi\left(x^{\prime}\right)\right) z^{\prime}+\left(3 x^{\prime}+\rho\left(x^{\prime}\right)\right) z^{\prime 2}+b x^{\prime}{z^{\prime}}^{3} \\
& +\left( \pm \frac{5}{\sqrt{10} \varepsilon}+\nu\left(x^{\prime}\right)\right) z^{\prime 4}+R^{\prime}, \quad \operatorname{ord}_{z^{\prime}} R^{\prime} \geqq 5,
\end{aligned}
$$

with ord $\psi \geqq 1$, ord $\rho \geqq 2$, ord $\nu \geqq 1$.

Consequently we have :

1) The indicds of $q$ and $q^{\prime}$ are the same. 
2) The positive side at $q$ is opposite to the positive side at $q^{\prime}$ with respect to $S_{1,1}(\bar{f})$

This proves the second part.

Consider mapping $\overline{\bar{f}}$ given by

$$
X=x, \quad Y=y, \quad Z=y z-z^{3}+\sigma(x) \sigma(y) \beta(z-x) .
$$

Then the above argument shows that the singularities of $\overline{\bar{f}}$ have the same properies as of $f$ except that the indices of $\overline{\bar{f}}$ are opposite to those of $f$. This proves the last part.

4. Topological consideration. We suppose that $f: M^{3} \rightarrow R^{3}$ is a generic decomposable mapping, and $M^{3}$ is an orientable closed smooth manifold. By definition there exist a locally homeomorphic mapping $i$ and a projection $\pi$ of $R^{4}$ to $R^{3}$ such that $\pi i=f$.

We may take a vector field $\left\{V_{p}\right\}$ on $R^{4}$ such that these vectors are projected to the null vector by $d \pi$. Since $i$ is an immersion, the differential of $i$ is an into-isomorshism from the tangent space of $M^{3}$ to that of $R^{4}$. Let $p$ be a point of $S_{1}(f)$ then $\operatorname{di}\left(T_{p}\right)$ contains the vector $V_{i(p)}$ where $d i$ is the differential of $i$ and $T_{p}$ is the tangent space of $M^{3}$ at $p$. Define now $\widetilde{V}_{p}=(d i)^{-1}\left(V_{i(p)}\right)$, then $\left\{\widetilde{V}_{p}\right\}$ is a smooth vector field on $S_{1}(f)$ which is contained in the null space $N(p)$. This vector field is called the null vector field.

Lemma 9. Let $f$ be a generic mapping. Then the connected components of $S_{1}(f)$ are orientable closed 2-manifolds.

Proof. Since $f$ is generic, $S_{1}(f)$ is a closed submsnifiold of $M^{3}$. Since the local degree of $f$ can be defined at points of $M^{3}-S_{1}(f)$, it follows that the normal bundle of $S_{1}(f)$ is trivial. Hence the lemma is proved.

Lemma 10. Let $f$ be a generic mapping and $D$ be a connected component of $S_{1}(f)$. Then we may take, in a neighborhood $U$ of $D$, a good 2-approximation $\bar{f}$ of $f$ such that the singularity $S_{1,1}(\bar{f}) \cap U$ is a connected set.

Proof. Let $E_{1}, \cdots, E_{i}$ be the singularities $S_{1,1}(f)$ in $D$, and $p$ be a point of $E_{1}$. We may take $E_{j}$ and a point $q \in E_{j}$ such that the points $p$ and $q$ are connected by a curve on $D$ without touch- 
ing any other point of $S_{1,1}(f)$. By Lemma 8, we may take in neighborhoods of $p$ and $q$ a deformation $f^{\prime}$ of $f$ such that the indices of the points of $S_{1,1,1}\left(f^{\prime}\right)$ near $p$ and $q$ are different. Then, by Theorem 1 there exists a deformation $f^{\prime \prime}$ of $f^{\prime}$ in $U(C)$ so that $E_{1}$ and $E_{j}$ are connected in the singularities $S_{1}\left(f^{\prime \prime}\right)$. By making such deformations successively, we obtain the lemma.

Remark. The decomposabllity of mapping is invariant under deformations if their first partial derivatives are close enough. The deformations in Section 3 are such deformations. Hence we may suppose that if the mapping $f$ in the last lemma is decomposable then so is $\bar{f}$.

Lemma 11. Suppose that the mapping $f$ is a generic decomposable mapping. Let $D$ a connected component of $S_{1}(f)$. If $S_{1,1}(f)$ $\cap D=E$ is a connected set then $E$ divides $D$ into two connected parts.

Proof. Let $p$ be a point of $E$. Then we may suppose that the singularities of $f$ is represented in a neighborhood of $p$ as follows :

$$
S_{1}(f): y-3 z^{2}=0, \quad S_{1,1}(f): y=z=0 .
$$

If $E$ does not divide $D$, we may take a simple closed curve $C$ in $S_{1}(f)$ so that $C$ is the intersection of $S_{1}(f)$ and $x=0$ in a neighborhood of $p$ and so that $C$ intersects with $E$ at a single point $p$. Take an orientation in $C$. Let $T_{r}$ be the tangent vector of $C$ at $r$ and $N_{r}$ be the normal vector of $C$ in the tangent plane of $S_{1}(f)$ at $r$. If $r$ is a point of $S_{1}(f)-S_{1,1}(f)$, then $\left\{T_{r}, N_{r}, \tilde{V}\right\}$ is a non-degenerate frame.

Take points $p^{\prime}=\left(0, \varepsilon, \sqrt{\frac{\varepsilon}{3}}\right), p^{\prime \prime}=\left(0, \varepsilon,-\sqrt{\frac{\varepsilon}{3}}\right)$ on $C$ for small $\varepsilon>0$, and consider these frames at $p^{\prime}$ and $p^{\prime \prime}$. We may suppose teat $\tilde{V}_{p}=\left(\frac{\partial}{\partial z}\right)_{p^{\prime}}, \quad \widetilde{V}_{p^{\prime \prime}}=\left(\frac{\partial}{\partial z}\right)_{p^{\prime \prime}}, N_{p^{\prime}}=\left(\frac{\partial}{\partial x}\right)_{p^{\prime}}$ and $N_{p^{\prime \prime}}=\left(\frac{\partial}{\partial x}\right)_{p^{\prime \prime}}$. Since the $y$-component of $T_{p^{\prime}}$, and $T_{p^{\prime \prime}}$ are opposite, the frames $\left\{T_{p^{\prime}}, N_{p^{\prime}}, \widetilde{V}_{p^{\prime}}\right\}$ and $\left\{T_{p^{\prime \prime}}, N_{p^{\prime \prime}}, \widetilde{V}_{p^{\prime \prime}}\right\}$ have opposite orientations. This contradicts to the orientability of $M^{3}$. This completes the proof. 
Lemma 12. Suppose that the mapping $f$ is a generic mapping. Let $p$ and $q$ be points of $S_{1,1,1}(f)$ which are contained in a connected component of $S_{1,1}(f)$. Suppose that there is no point of $S_{1,1,1}(f)$ between $p$ and $q$. Then the following two cases occur.

1) The positive sides at $p$ and $q$ are the same side with respect to $S_{1,1}(f)$ in $S_{1}(f)$, and the indices of $p$ and $q$ are different.

2) The positive sides at $p$ and $q$ are opposite with respect to $S_{1,1}(f)$ in $S_{1}(f)$, and the indices of $p$ and $q$ are the same.

Proof. Let $C$ be an open oriented subarc of $S_{1,1}(f)$ between $p$ and $q$. There exist coordinate systems at $p$ and $q$ under which $f$ is represented in the form in the sence of Section 1 . Take in a tubular neighborhood of $C$ a Riemannian metric which induces Euclidean metric determined by the coordinate systems at $p$ and $q$. Let $v_{1}(p), v_{2}(p)$ and $v_{3}(p)$ denote respectively the tangent vectors of $x-, y$ - and $z$-axis in the coordinate system at $p$. For $q$, use the same notation.

Let $s$ be a point of $C$. Let $T(s)$ denote the tangent vector of $S_{1,1}(f)$ at $s, N(s)$ the null space at $s$, and $W(s)$ the normal vector to $S_{1}(f)$. The orientation of $W(s)$ is determined by the direction from the negative side to the positive.

Let $r$ be a point of $C$ near $p$, and give $N(r)$ the orientation determined by the diredtion of $z$-axis in the coordinate system at $p$. Then the plane $(W(r), N(r))$ converges to the plane $\left(v_{1}(p)\right.$, $\left.v_{3}(p)\right)$ if $r$ converges to $p$. Hence we can compare the orientation of $\{W(r), N(r)\}$ with that of $\left\{v_{1}(p), v_{3}(p)\right\}$. We divide two cases according as the $z$-component of the coordinate of $r$ is negative or positive.

Negative case: In this case, the directions of $N(r)$ and of $v_{2}(p)$ are the same in $S_{1}(f)$ with respect to $S_{1,1}(f)$, and the orientations of $\{W(r), N(r)\},\left\{v_{1}(p), v_{3}(p)\right\}$ are opposite.

Positive case: In this case the directions of $N(r)$ and of $v_{2}(p)$ are opposite in $S_{1}(f)$ with respect to $S_{1,1}(f)$ and the orientations of $\{W(r), N(r)\}$ and of $\left\{v_{1}(p), v_{3}(p)\right\}$ are the same.

For $r$ near $p, N(r)$ is already oriented. These determine naturally the orientation of $N(s)$ for any $s \in C$. Then $\{\{W(s), T(s)$, 


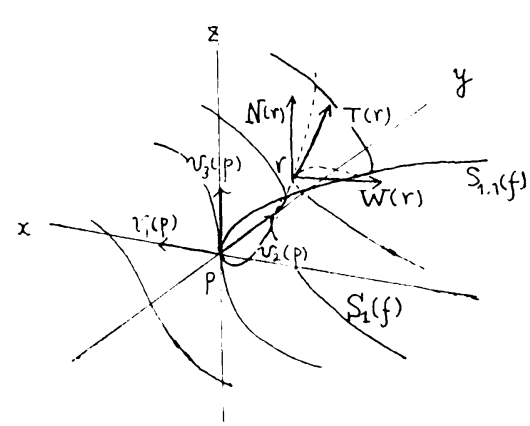

Negative Case

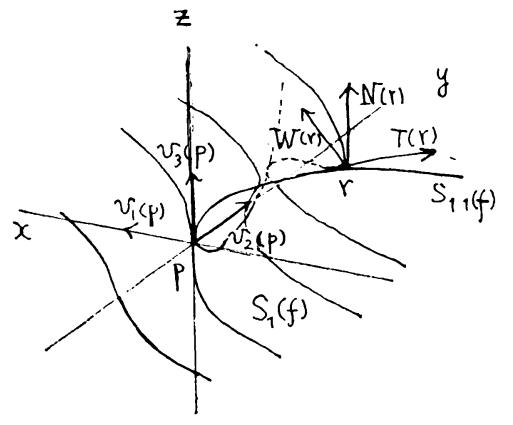

Positive Case

$N(s)\}, s \in C\}$ is a continous family of non-degenerate frames. We may suppose that the direction of $z$-axis in the coordinate system at $q$ is the same to that of $N(t)$ for a point $t$ near $q$. Compare now the orientations of $\left\{v_{1}(p), v_{2}(p), v_{3}(p)\right\}$ and of $\left\{v_{1}(q), v_{2}(q)\right.$, $\left.v_{3}(q)\right\}$. It occurs two cases according as the directions of $v_{2}(p)$ and $v_{2}(q)$ are the same or not in $S_{1}(f)$ with respect to $S_{1,1}(f)$.

Case I (The directions are the same). In this case, if the directions of $v_{2}(p)$ and of $N(r)$ are the same with respect to $S_{1,1}(f)$ then the directions of $v_{2}(q)$ and of $N(t)$ are the same with respect to $S_{1,1}(f)$. Hence the above negative cases arises for $(p, r)$ and $(t, q)$. Therefore it follows that the orientations of $\left\{v_{1}(p), v_{2}(p)\right.$, $\left.v_{3}(p)\right\}$ and of $\{W(r), T(r), N(r)\}$ are opposite, and that the orientations of $\{W(t), T(t), N(t)\}$ and of $\left\{v_{1}(q), v_{2}(q), v_{3}(z)\right\}$ are the same.

If the directions of $v_{2}(p)$ and of $N(r)$ are opposite in $S_{1}(f)$ with respect to $S_{1,1}(f)$, then the directions of $v_{2}(q)$ and of $N(t)$ are opposite in $S_{1}(f)$ with respect to $S_{1,1}(f)$. Thus the above positive case arises for $(p, r)$ and $(t, q)$.

Hence we have that the orientations of $\left\{v_{1}(p), v_{2}(p), v_{3}(p)\right\}$ and of $\{W(r), T(r), N(r)\}$ are the same, and that the orientations of $\{W(t), T(t), N(t)\}$ and of $\left\{v_{1}(q), v_{2}(q), v_{3}(q)\right\}$ are opposite.

As a consequence of the argument above it follows that the indices of $p$ and of $q$ are different.

Case II (The directions are opposite). In this case, the similar consideration shows that the indices of $p$ and of $q$ are the same.

Corollary. Let $f$ be a generic mapping of $M^{3}$ tn $R^{3}$, where $M^{3}$ 
is an orientable closed smooth manifold. Let $C$ denote a connected component of $S_{1,1}(f)$. Then the number of points of $S_{1,1,1}(f)$ in $C$ is even.

Theorem 2. Let $M^{3}$ be an orientable closed smooth 3-manifold and $f$ be a mapping of $M^{3}$ to $R^{3}$. Suppose that $f$ is a generic decomposable mapping. Then we may take a good 2-approximation $\bar{f}$ of $f$ so that $S_{1,1,1}(\bar{f})$ is empty and $S_{1,1}(\bar{f})$ are boundaries of domains of $S_{1}(\bar{f})$.

Proof. By Lemma 10, we may suppose that the part $E$ of $S_{1,1}(f)$ which is contained in a connected component $D$ of $S_{1}(f)$ is connected. Thus, by Lemma $11, E$ divides $D$ into two domains. Now let $p$ and $q$ be points of $S_{1,1,1}(f) \cap E$ between which there is no point of $S_{1,1,1}(f)$.

Case 1 (The indices of $p$ and $q$ are different). In this case, the positive sides at $p$ and at $q$ are the same side with respect to $E$. Now we may consider the curve $C$ running from $p$ to $q$ whose interior is contained in $D-E$ and which starts from $p$ into the negative side and which ends to $q$ from the negative side. Then, by Theorem 1 , we may eliminate $p$ and $q$ from $S_{1,1,1}$.

Case 2 (The indices of $p$ and $q$ are the same). In this case, the positive sides at $p$ and at $q$ are opposite side with respect to $E$. Let $r$ be a point of $E$ between $p$ and $q$. By Lemma 8, we may take in a small neighborhood of $r$ a good 2-approximation $f^{\prime}$ of $f$ so that there exist, between $p$ and $q$, two new point $r^{\prime}$ and $r^{\prime \prime}$ of $S_{1,1,1}\left(f^{\prime}\right)$ whose indices are different from those of $p$ and $q$. Then applying the same method as in case 1 to $\left(p, r^{\prime}\right)$ and $(q, r)$, it follows that we may eliminate $p$ and $q$ from $S_{1,1,1}$.

The above argument shows that there exists an approximation $\bar{f}$ of $f$ such that $S_{1,1,1}(\bar{f})$ is empty.

It is easily shown that each connected component of $S_{1,1}(\bar{f})$ is the boundary of a 2-disk or of a domain in $S_{1}(\bar{f})$.

Lemma 13. Let $E^{\prime}$ be a smooth circle in $M^{3}-S_{1}(f)$, and suppose that $E^{\prime}$ is the boundary of an orientable smooth 2-manifold $D^{\prime}$ in $M^{3}-S_{1}(f)$. Then we may take in a neighborhood $U\left(D^{\prime}\right)$ of $D^{\prime}$ a 
good 0-approximation $f^{\prime}$ of $f$ so that the mapping $f^{\prime}$ is a generic mapping and $S_{1,1}\left(f^{\prime}\right) \cap U\left(D^{\prime}\right)=E^{\prime}$ and $S_{1,1,1}\left(f^{\prime}\right) \cap U\left(D^{\prime}\right)=\varnothing$.

Proof. For a given $D^{\prime}$, there exist sets $D_{1}, D_{2}$ such that $D_{1} \varsubsetneqq$ $D^{\prime} \risingdotseq D_{2}, D_{2} \cap S_{1}(f)=\varnothing$ and $D_{2}-D_{1}$ is diffeomorphic to $E^{\prime} \times[-1,1]$. Then we may take a neighborhood $U\left(D^{\prime}\right)$ of $D^{\prime}$ which is diffeomorphic to $D_{2} \times[-1,1]$ and which is contained in $M^{3}-S_{1}(f)$.

Take a smooth function $\rho(x, t)$ having the following properties :

1) $\rho(x, 0)=x$, for $|x| \leqq 1$,

2) $\frac{\partial \rho}{\partial x}(x, t)>0$ for $0 \leqq t<\frac{1}{2}, \rho(x, t)=x$ for $1 \geqq|x| \geqq \frac{2}{3}$,

3) $\rho(x, t)=(4 t-1) x^{3}+(-2 t+1) x$, for $\frac{1}{2} \leqq t \leqq 1,|x| \leqq \frac{1}{3}$,

$$
\begin{aligned}
& \rho(x, t)=x \text { for } \frac{1}{2} \leqq t \leqq 1,1 \geqq|x| \geqq \frac{2}{3}, \\
& \frac{\partial \rho}{\partial x}(x, t)>0 \text { for } \frac{1}{2} \leqq t \leqq 1,1 \geqq|x|>\frac{1}{3} .
\end{aligned}
$$

We may take a smooth function $\nu(p)$ on $D_{2}$ such that

$$
\begin{aligned}
& \nu(p) \geqq 0 \text { for } p \in D_{2}, \quad \nu(p)=1 \text { for } p \in D_{1}, \\
& \nu(p)=\frac{1}{2} \text { if and only if } p \in E^{\prime}, \\
& \nu(p)=0 \text { for } p \in \partial D_{2} .
\end{aligned}
$$

Then we have a smooth mapping $h$ of $U\left(D^{\prime}\right)$ to $U\left(D^{\prime}\right)$ defined by

$$
h(p, x)=(p, \rho(x, \nu(p)))
$$

where $p \in D_{2}, x \in[-1,1]$.

Since the mapping $h$ is the identity on the boundary of $U\left(D^{\prime}\right)$, $h$ has an extension $h^{\prime}: M^{3} \rightarrow M^{3}$ so that $h^{\prime} \mid M^{3}-U\left(D^{\prime}\right)=$ the identity.

It is now easily shown that the mapping $f^{\prime}=f h^{\prime}$ satisfies the conditions of the lemma.

Theorem 3. Let $M^{3}$ be an orientable closed smooth manifold, and $f$ be a generic decomposable mapping of $M^{3}$ to $R^{3}$. Then we may take a good 0-approximation $\bar{f}$ of $f$ so that $S_{1,1}(\bar{f}) \neq \varnothing$.

Proof. By Theorem 2, we may suppose that $S_{1,1,1}(f)$ is empty and any circle of $S_{1,1}(f)$ is the boundary of a domain of $S_{1}(f)$. Denote by $E$ one of the components of $S_{1,1}(f)$. By Lemma 7, we 
may take a coordinate system $(x, y, z)$ in a neighborhood of $E$ and the parameter system $(X, Y, Z)$ in $R^{3}$ so that a good 3-approximation $f^{\prime}$ of $f$ is represented by

$$
X=x, \quad Y=y, \quad Z=y z-z^{3} .
$$

Let $E^{\prime}$ be the set of points $(x,-\varepsilon, 0)$ where $\varepsilon>0$ is sufficiently small. Then $E^{\prime}$ satisfies the conditions of Lemma 13, and hence we may take a good 0-approximation $f^{\prime \prime}$ of $f^{\prime}$ which is represented by the following equations in a tubular neighborhood of $E$ :

$$
X=x, \quad Q=y, \quad Z=\rho(y) z-z^{3},
$$

where $\rho(y)$ is a smooth function which has the following conditions :

$$
\begin{gathered}
\rho(y)=0 \text { for } y=0,=\varepsilon \\
\rho(y)<0 \text { for } 0<y<\varepsilon \\
\frac{d \rho}{d y}(0)<0, \quad \frac{d \rho}{d y}(\varepsilon)>0 .
\end{gathered}
$$

Applying Lemma 1 to each section : $x=$ constant, we obtain a good 0 -approximation $f^{\prime \prime}$ of $f^{\prime}$ so that $E$ is eliminated from the singularities $S_{1,1}$. By this methods we may obtain a good 0-approximation $\bar{f}$ which satisfies the condition in the theorem.

Theorem 4. Let $M^{n}$ be an orientable closed smooth $n$-manifold and $f$ be a mapping of $M^{n}$ to $R^{n}$. Suppose that the singularities of $f$ satisfy the following conditions:

$$
S_{i}(f)=\varnothing \quad(i \geqq 2), \quad S_{1,1}(f)=\varnothing .
$$

Then the mapping $f$ is decomposable mapping.

Proof. By the condition $S_{i}(f)=\varnothing(i \geqq 2), S_{1}(f)$ is an $(n-1)$ dimensional smooth submanifold of $M^{n}$. Since $S_{1,1}(f)=\varnothing$, it follows that $f \mid S_{1}(f)$ is a local homeomorphism. The null space $N(p)$ is transversal to $S_{1}(f)$ because of $S_{1,1}(f)=\varnothing$. Since $M^{n}$ is orientable, we may define the local degree of $f$ at points of $M^{n}-S_{1}(f)$. Hence the normal bundle of $S_{1}(f)$ is trivial, and we may take an orientation in $N(p)$ so that $\left\{N(p) ; p \in S_{1}(f)\right\}$ is a transversal vector field. Denote by $L_{p}$ the geodesic segment whose tangent vector 
at $p$ is $N(p)$ Then $U=\bigcup_{p \in S_{1}(f)} L_{p}$ is a neighborhood of $S_{1}(f)$. Now we may take a smooth function $g(q)$ on $U$ such that the derivative of $g(q)$ with respect to the vector $N(p)$ is not zero. For example, we may take as $g(q)$ the length of $L_{p}$ from $p$ to $q$. Then the function $g(q)$ can be extended to a smooth function $g$ on $M^{n}$. Denote $h(p)=(f(p), g(p))$, then $h$ is a smooth mapping of $M^{n}$ to $R^{n+1}$ which is a local homeomorphism.

Thus the theorem is proved.

\section{REFERENCES}

[1] A. Haefliger, Quelques remarques sur les applications differentiables d'une surface dans le plan, Ann. de L'institut Fourier, 10 (1960), 47-60.

[2] R. Thom, Quelques propriètés globales des variètés différentiables, Commentarii Math. Helvetici, 28 (1954) 17-86.

[3] R. Thom, Les singularités des applications différentiables, Ann. de L'institut Fourier, 6 (1956) 43-87.

[4] H. Whitney, On singularities of mappings of Euclidean spaces, I, Mappings of the plane into the plane, Ann. of Math. 62 (1955) 374-410.

[5] H. Whitney, Singularities of mappings of Euclidean spaces, Sympos. Int. de Topologia Algebraica, 285-301. 